\title{
Design of a Centralized Intelligent Expert System and Contamination Detection of Tissue Cultured Sugarcane Crop
}

\author{
Mujahid Hussain Memon ${ }^{1,2}$, Arif Karim, Dr$^{1}$. Waheeduddin Hyder ${ }^{2}$, Fayaz Ali
}

Dharejo $^{3}$, Munsif Ali Jatoi ${ }^{4}$, Dr. Beena Naqvi ${ }^{1}$, Faisal Ghazanfar ${ }^{1}$, Farhan Aziz ${ }^{1}$

\begin{abstract}
The paper presents a design of cloud based IoT enabled smart agriculture application for HiTech tissue cultured sugarcane crop entitled "Design of Centralized Intelligent Expert System and Contamination Detection of Tissue Cultured Sugarcane Crop". This expert system comprises of Raspberry Pi-4 (RPi), Arduino-Mega, GSM-Modem (Sim900) and sensormodules for monitoring and control of essential parameters of growth-rooms inside a tissue cultured laboratory. The parameters monitored are temperature, humidity and light intensity with artificial day light timing and control. Moreover, AI-based health prediction suggests the detection of contamination of sugarcane crop, which has been applied at edge device. In addition, fire-smoke sensor and methane gas sensor are incorporated for fire protection and any disastrous situation. Three numbers of webcams are attached to the RPi for monitoring growth and health of explants. This is to get meaningful insights of data for future decision making in maximizing crop yield and quality. An AI-Model was developed for detection of contamination that predicts the health of Tissue Cultured Sugarcane Crop, which has also the beauty of image enhancement which is covered applying Generative Adversarial Networks (GAN)". In this system, the RPi reads sensor's data through Arduino and convert it to dataframe with timestamp and geo-tag. The data along with the captured images are sent to a centralize cloud application for applying Data Mining and Artificial Intelligence. Due to the great need of sugarcane crop in Pakistan, the Plant Tissue Culture (PTC) technology has been incorporated with AI. The proposed system is aimed to be installed at established PTCgrowth-rooms for sugarcane crop so that domain experts can be connected to the cloud application for its monitoring, control and data analytics. In addition, the use of telepresence through cloud application will enable PTC-experts to provide assistance to the remote user and resolve their issues timely, thus extending PTC technology all over the country which will eventually lead to increase crop yield with quality in affordable price.
\end{abstract}

${ }^{1}$ Pakistan Council of Scientific and Industrial Research (PCSIR) Laboratories Complex Karachi, Pakistan.

${ }^{2}$ Ilma University, Karachi, Pakistan.

${ }^{3}$ University of Chinese Academy of Sciences, Haidian, Beijing, China.

${ }^{4}$ Department of Biomedical Engineering Salim Habib University, Karachi, Pakistan. SJET | P-ISSN: 2616-7069 |E-ISSN: 2617-3115 | Vol. 4 No. 2 July - December 2021 
Keywords: Artificial Intelligence, Deep Learning, Internet of Things, Tissue Culture, Sugarcan

\section{Introduction}

Plant Biotechnology is emerging domain with excessive scope in our country. In Plant Tissue Culture (PTC) which is a subdomain of biotechnology, has very complex and dynamic environment for monitoring and control the growth of tissue cultured plants. The vital parameters to monitor and control are temperature, humidity and light intensity of the tissue culture growth room with artificial daylight timings. In addition, methane gas sensor and fire-smoke sensor are incorporated for fire protection and to avoid any mishap. Three numbers of webcams are attached to the RPi for monitoring growth and health of explants. Similarly, to predict the health of cultured plants, it has been a big challenge to detect disease of infected plants, where Deep Learning (DL) and Machine Learning (ML) methods have been utilized by researchers in agriculture sector for crop monitoring. This field has a potential need of utilization of ICT due to the climate changes and biodiversity and involvement of big-data generated.

The fundamental idea of proposed work entitled: "Design of Centralized Intelligent Expert System and Contamination Detection of Tissue Cultured Sugarcane Crop" is to predict the health of crop and facilitate the domain experts (PTC-Experts) to collaborate and assist the remote users to mitigate the day to day issues. The work is also an effort to transform agriculture sector into Industry 4.0.

Inside Laboratory, small pieces of explants of sugarcane are used under controlled environment. The culture is initiated in 20 to 30 days on nutrient media with and without growth regulator. The growth of ex-plant depends upon maintenance of aseptic and controlled conditions e.g. temperature, humidity and light. After initiation of culture, the explants are subculture on multiplication media for multiplication of explant and later on incubated for 20 to 25 days under controlled environment. Approximately 5 to 6 times subcultures are utilized for multiplication from a single explant. Tissue culture plants are healthy, disease free and high yielding product.
In order to get a huge amount of crop, there would be a need of several tissue culture growth rooms but due to scarcity of the scientific staff (domain experts), they cannot manage their visits for monitoring the cultured plants.

To address this issue, a large numbers of cultured sugarcane growth rooms can be monitored remotely by sitting at a centralized point using a cloud-based application. Keeping in view to utilize such expert system, Artificial Intelligence (AI) plays an effective role for health prediction of cultured plants. AI's subdomain Deep Learning (DL) wrapper eases the design, training and development of models over many frameworks such as Theano, Tensor Flow, Torch, Chainer, and Keras. These frameworks decrease the efforts of AI engineers in DL but still the training of the model is not a piece of cake; it needs a huge amount of data. Our proposed methodology is the utilization of Cloud based Expert System and Health Prediction of Cultured Plants using Deep Neural Networks (DNN) aiming to monitor and making a system to take actions timely and virtual visits be performed using telepresence.

\subsection{Plant Tissue Culture Technology}

It is an emerging field which has a great potential and scope in agriculture industry for studying and solving basic and applied problems in the field of plant biotechnology. For mass production of crop, the Micropropagation and other In-vitro techniques are widely used in commercial horticulture and agricultural [1]. A small piece of a plant either from root, shoot, leaf, and even a single cell is cultured in an aseptic and controlled environment on nutrient media supplemented with or without growth regulator. This isolated small piece of plant is termed as explant. Under controlled temperature, humidity, and light conditions the explant is micro-propagated to form a clone of the mother plant [2]. The explants after getting reasonable growth are transferred to the semi-controlled conditions in green house for acclimatization. Healthy and disease-free plants are selected for tissue 
culture. The crop yield and quality can be improved through tissue culture technique.

In a continuous production environment, there should be multiple tissue culture labs near the actual growth site to develop good quality seeding material to get better yield. For mass production, a large numbers of PTC labs would be required in the remote areas along with designated PTC experts. The monitoring and control of temperature, humidity and light conditions are required for tissue culture lab to maintain a healthy culture in growth room. It will be impractical to monitor these conditions manually on large scale either due to shortage of skilled experts or frequently traveling and visiting PTC sites.

\subsection{Tissue Cultured Sugarcan}

Sugarcane (Saccharum officinarum L.) is a vivid member of Poaceae family is a perennial grass. It is normally propagated in two modes either by seeds or vegetatively. It is vegetatively propagated by stem cutting, having two or three buds called sets, whereas advanced approaches belonging to In-vitro propagation was done by using apical or axillary bud and young leaf as an explant. The tissue culture of sugarcane from different regions of the world was not properly reported but the first published attempt to regenerate sugarcane crop through In-vitro technique was made by Heniz and Mee (1969) [1]. For the breeding purpose, it is also reproduced through seed propagation via flower (fuzz) [3]. Sugarcane Breeding Institute (SBI) [4] is responsible for the development of its varieties, hence it is obtained after breeding which was raised from fuzz.

In order to provide the quality production of sugarcane crop, the development of tissue cultured sugarcane crop for quick multiplication of disease-free yield is a significant step towards the field of biotechnology. For most micropropagation work the explant of choice is an apical or an axillary bud. Usually, the explants are more responsive to culture treatments if they are collected during the period of active growth [5]. For sugarcane micropropagation, the best explant type tissue cultured sugarcane crop is shoot tips which enhance the growth of sugarcane Top actively [6]. Shoot tip of sugarcane which can be usually obtained from 3 sources: Top of the actively growing cane, elongating axillary shoot from decapitated shoots and dormant axillary buds [1]. Usually the flowers are not feasible for any technique due to its high variability. The availability of high quality and true-type-planting material of fresh varieties of sugarcane is a great challenge which poses constraint in rapid acceptance for commercial use. Even well-accepted profitable and commercial varieties cultivation require an excellent seed to guarantee the better cane yield, sugar yield with disease-free crop[7]. Furthermore, the traditional methods of cultivation uses three-budded set which always requires a large quantity of seeds, thus, costly, time-consuming and less productive; only 5 to 10 canes per seed. On the other hand, a tissuecultured seed can produce 30 to 50 canes [8].

In leading agricultural countries of AsiaPacific region like Australia, India, and the Philippines have already applied this technique for commercial use and these countries have been getting more benefits through rapid propagation and distribution of elite varieties. The development of tissue culture technology for rapid multiplication of disease-free planting material has been an important step towards high quality and mass production [9]. Moreover, PTC techniques deliver an alternative process for multiplication and improvement of sugarcane crops [10]. In a short period and faster rate, micropropagation of cane for quality and phytosanitary planting material, PTC offers the best methodology. As compared to the traditional seed techniques, PTC technique can guarantee to increase the production by 20-35 times [11]. From one shoot a large number of plants are produced using the technique of micropropagation. These were mandatory as compared to 8.8 tons of cane seed in conventional methods for planting in an area of a hectare. Thus, the multiplication ratio was 100-150 times using PTC as matched to 11-12 using conventional cane sets, prominent to a drastic reduction in seed cane prerequisite [12] Kuar \& Sandhu [13] have experimented and worked on the shoot multiplication rates which were ranged 
from 4 to 25 fold and resultantly with $97 \%$ survival rate, the complete plantlets were achieved in 157 days.

\subsection{Culture Contamination}

In PTC Techniques, the contamination is the presence of undesirable element that spoils cultured crop. Contamination in tissue culture is originated from two sources, 1) the carryover of microorganisms in the tissues of explants or on its surface, 2) following inappropriate SOP's inside the laboratory [14]. In the sequence of plant production and its growth, the microorganisms may move in the tissues of the plant through a natural setting. However, where explants appear uncontaminated, it may be necessary to bulkup the material to provide adequate material for testing [15]. Plants may thus develop or cultivate endophytic 'floras' of variable species composition consisting of Inter and Intracellular microorganisms including Viroids, Viruses, Prokaryotes (bacterial and bacteria-like agents), and Fungi. In establishing tissue cultures, subject on the explant consumption for propagation, possibly surface and endophytic microorganisms are carried over into culture. It must be mentioned here that the detection of contamination is the core research work of our study, where image processing with DNN would be studied and an AI-Model would be developed in order to predict the health of tissue culture sugarcane crop.

\section{Previous Work}

The work of Pezhman Taherei Ghazvinei and et al. [16] using ML and ANN presented "Sugarcane growth prediction based on meteorological parameters", where the parameters of the crop were obtained as data by field trials of selected sugarcane variety. Their experiment is based on data regarding the comparative study of daily sugarcane growth within accumulative daily irrigation water.

Aman and et al. [17] presented "Smart Agriculture Monitoring System using IoT" based on "Thingspeak" and "ARM7". They monitored humidity, temperature and soil- moisture of the land used for the plants. He has worked on IoT for analyzing the agricultural operations where he has proposed to get data of parameters like atmospheric, temperature and soil fertility, so the farm condition can be monitored remotely. Although, the work of Dandy and et al. [18] is only focused on temperature and humidity monitoring in tissue culture laboratory for banana crop. They have designed a prototype using Arduino-Uno for overall control and GSM modem for SMS notification. He has termed the monitoring of temperature and humidity to maintain the fast growth and resistant to diseases and infection. Traditionally, Abeer A. Elsharif and Samy S. Abu-Naser [19] have worked on "An Expert System for Diagnosing Sugarcane Diseases" using CLIPS and Delphi languages they have developed a standalone desktop application and few signs have been described for selection, where one has to select the symptoms of a disease; hitherto no automation and AI was applied.

Similarly, Noor Hafizahand and et al. [20] have also presented a web-based system for monitoring of tissue culture growth rooms and send emails and SMS alerts. Sensors are also incorporated with database to monitor critical parameters for data analysis.

The work of Bashar Alhnaity and et al. [21] utilized deep learning for tomato plant growth and yield prediction in controlled greenhouse environment. The AI model utilized is a new Deep RNN (Recurrent Neural Network) with LSTM (Long Short-Term Memory) neuron model that targeted growth parameters applied on time series data. Similarly, the work of Mohsen Hesami and et al. [22] incorporated Support Vector Machine (SVM) with Genetic Algorithm for modeling PTC procedures especially the effect of growth regulators on explants in embryogenesis. In another work ANN (Artificial Neural Network) has been used to predict media composition for callus growth [23]. A similar effort was also made by Anna and et al. [24] after incorporating ANN, Support Vector Regression (SVR), M5-prime Regression Trees, Random Forests (RF), and K-Nearest Neighbors for prediction of the crop. 
Mujahid Hussain Memon et al. Design of a Centralized Intelligent Expert System and Contamination Detection of

\section{Problem Statement}

Plant tissue culture a subdomain of biotechnology has a great potential in our country that needs an intelligent monitoring system in order to bring this diligence towards Industry 4.0 for vision 2025 applying Information \& Communication Technology (ICT) to boost economy of the country. There is great need of its utilization both in private and public sectors which may be a billion dollar industry in the future. This industry has been facing the scarcity of experts in Pakistan especially for monitoring systems of culturedplants growth rooms located remotely. The few experts cannot manage a number of laboratories / growth-rooms for monitoring and their frequent visits is almost impossible. In order to monitor growth-rooms, the parameters like temperature, humidity, light intensity, day-light timing, growth and health of explants is essential for an expert. Moreover, a remotely located laboratory also needs additional sensors for protection, i.e. detection of fire-smoke and flammable gases. Further, the growth-room needs temperature between 25 to $29^{\circ} \mathrm{C}$ depending on the type of explants. Similarly, the growth and health of plants are required to monitor visually by domain experts. The growth rooms also need $24 / 7$ electricity and alternate energy sources or a backed-up generator to keep the laboratory lightening and controlled temperature to run the system smoothly. The artificial lights are needed for a short-day period that is an alternative of a day light. Finally, the domain experts will be facilitated to analyze the data at a centralized cloud-based application and would be able to observe the growth of explants and future decision would be taken remotely to maximize the quality of production. The proposed system is named as Tissue Culture Laboratory Management System (TCLMS).

\section{Material and Methods}

Pakistan has a great potential of applying PTC in agriculture sector which will eventually lead to socioeconomic development. This technique has been successfully used for different crops such as sugarcane, banana, rice, tomatoes and potatoes in developed countries. After the establishment of a laboratory, the real challenge is to monitor the environment of growth-rooms and explant for health and contamination. The domain experts regularly visit these sites for monitoring and examining the explant so that corrective measures can be carried out. Meanwhile, they analyze collected data for getting results, however, their perception is far low from AI-based DSS (Decision Support System). This paper is intended to serve the industry of biotechnology with an intelligent system tissue culture growth rooms of sugarcane crop which can be connected to a centralize application via $\mathrm{WiFi}$ and GSMmodem, where PTC-experts will monitor the process remotely and perform required actions to run a number of growth room remotely. The Edge-Device (TCLMS) will send respective data and images to the cloud application. The attached 3nos. of cameras will send pictures of explants to the cloud as per configured sampling time. The environmental parameters are temperature, humidity, light intensity, daylight timing, fire-smoke and presence of methane gas. These parameters are essential for motoring the growth of explants and detection of any mishap situation to safeguard the laboratory.

\subsection{Hardware Architechture}

Hardware Architecture The hardware architecture for "Design of Centralized Intelligent Expert System and Contamination Detection of Tissue Cultured Sugarcane Crop" is shown in Fig. 7. There are two processing units, i.e. Raspberry Pi-4 (RPi) and ArduinoMega. RPi is responsible for gathering images from cameras (3nos.); connectivity through GSM \&WiFi; and finally acquisition of sensor's data from Arduino-Mega. The Arduino-Mega and cameras are connected to RPi through USB port. Similarly, the GSM modem (Sim900) is attached to the serial port of RPi as shown in Fig. 1. 


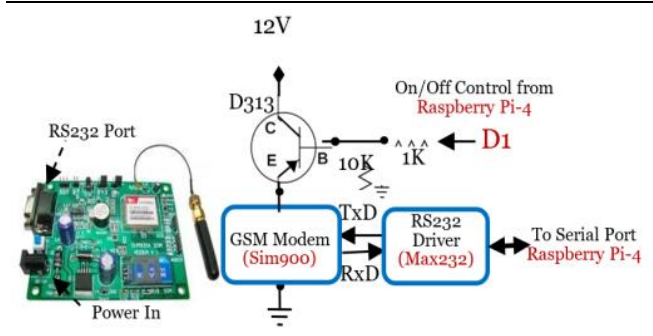

Fig.1. GSM Modem (Sim900) with on/off control circuit and RS232 driver circuitry for energy conservation.

The RPi is powered by rechargeable battery $(12 \mathrm{~V} \& 12 \mathrm{~A})$ with 5 Volt voltage regulator and charging circuitry. In case of power failure, the battery can run the TCLMS for $\sim 10$ hours. The second processing unit, i.e. Arduino is utilized for collecting sensor's data through $\mathrm{I}_{2} \mathrm{C}$ port. Sim900 module is connected through Max232 (serial driver IC) to serial port of RPi as shown in Fig.1. The power is supplied to Sim900 module through a transistor (D313) used for critical situation, i.e. when there is no WiFi connectivity. This module is incorporated due to its feature of sending text and connectivity of internet for laboratories located in remote areas of different parts of country. The sensors utilized are Methane / Natural Gas Sensor (MQ4), Temperature and Humidity Sensor (SHT-30), Light Sensor (GH170) and Fire/Smoke Sensor (ApolloXP95).

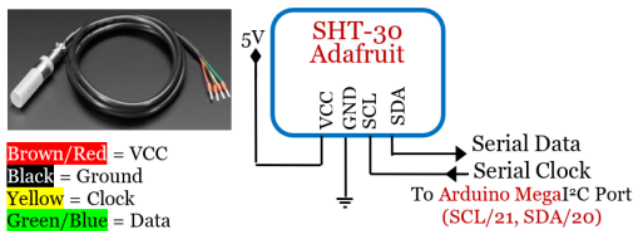

Fig. 2 Adafruit SHT-30 Sensor for Temperature \& Humidity measurement.

The temperature sensor incorporated is SHT-30 from Adafruit. It is water proof and mesh protected. The connectivity to Ardunio is established via $\mathrm{I}^{2} \mathrm{C}$ interface as shown in Fig.2.

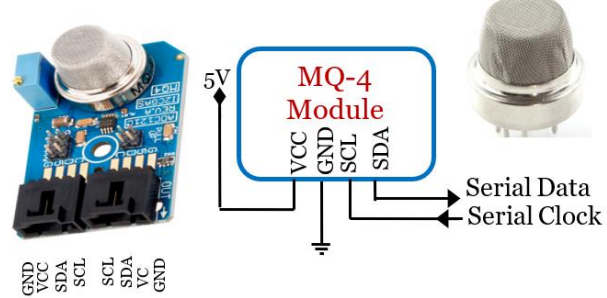

Fig. 3. MQ4 Methane/Natural Gas Sensor Module with 12-Bit $\mathrm{ADC}$ and $\mathrm{I}^{2} \mathrm{C}$ interface.

For fire protection, i.e. detection of flammable gases, a methane / natural gas senor (MQ-4 Module) is utilized. It is connected to Arduino via its $\mathrm{I}^{2} \mathrm{C}$ interface as shown in Fig.3. The detection range is 300 to $10000 \mathrm{ppm}$ with temperature working range of -10 to $50^{\circ} \mathrm{C}$.

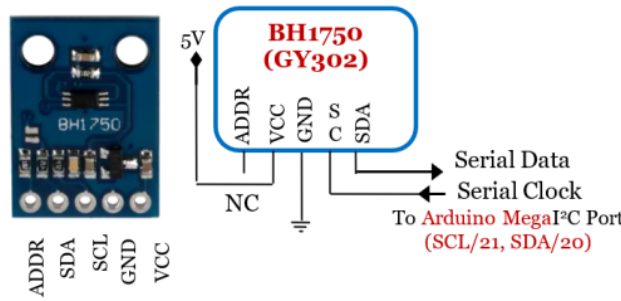

Fig. 4. GH1750 Ambient Light Sensor Module with I2C Interface.

$\mathrm{T}$ The ambient light intensity measurement with BH1750 module having range of $1 \sim 65535 \mathrm{Lux}$, is connected via $\mathrm{I}^{2} \mathrm{C}$ interface to the Arduino as shown in Fig.4. The average light requirement for a laboratory is 500 2000 Lux.
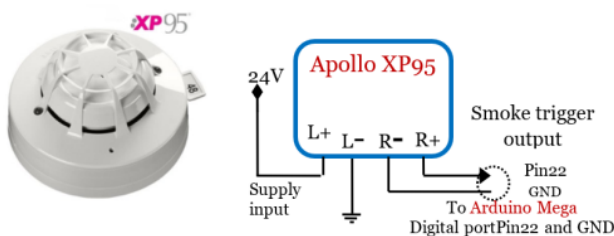

Fig.5. Fire Smoke Detector Module with connections

To prevent any mishap, the detection of flammable gases, fire and smoke detector (Apollo XP95) is also integrated to detect flame or smoke as shown in Fig.5. This will also send alerts via GSM-Modem, to the 
Mujahid Hussain Memon et al. Design of a Centralized Intelligent Expert System and Contamination Detection of

responsible person in order to take necessary actions.

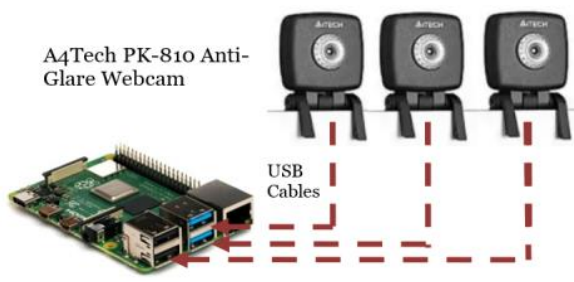

Fig. 6 Raspberry Pi-4 connected with 03nos. of Web-Cams

The integration of three nos. of webcams with RPi is shown in Fig.6. These webcams will motor different explants simultaneously in growth-rooms located remote location. Furthermore, to minimize the cost of servers, the images and sensors' data are stored in RPi memory which is later on send to the cloud storage as per scheduled time. The cloud data will be utilized for data mining.

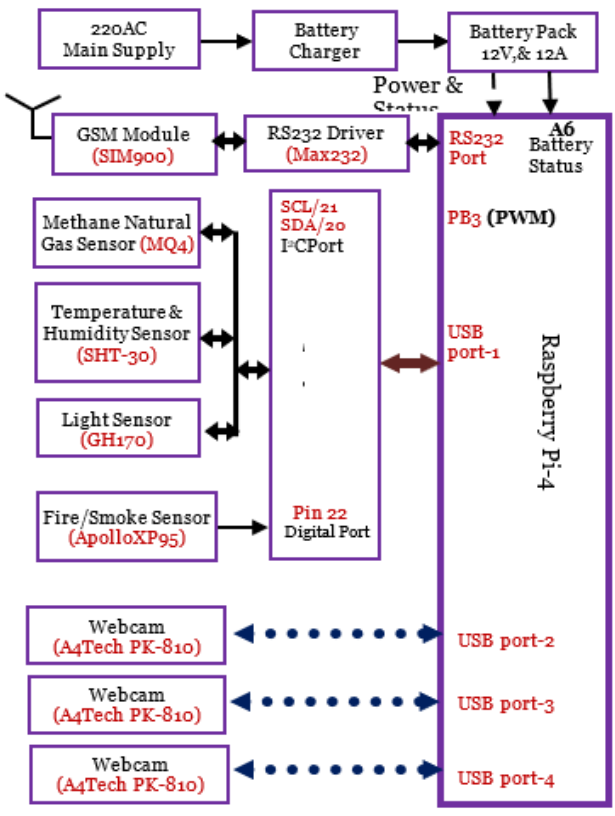

Fig. 7. Hardware Architecture of TCLMS

\subsection{Software Architechture}

There are two main processing units, i.e. $\mathrm{RPi}$ and Arduino Mega which requires embedded software application for monitoring, control and connectivity to the cloud application. Firstly, RPi is powered by Raspberry Pi OS (previously known as Raspbian). A software application in python is modeled as shown in Fig.8. Similarly, an application in C-Language is modeled for Arduino mega as shown in Fig.9.

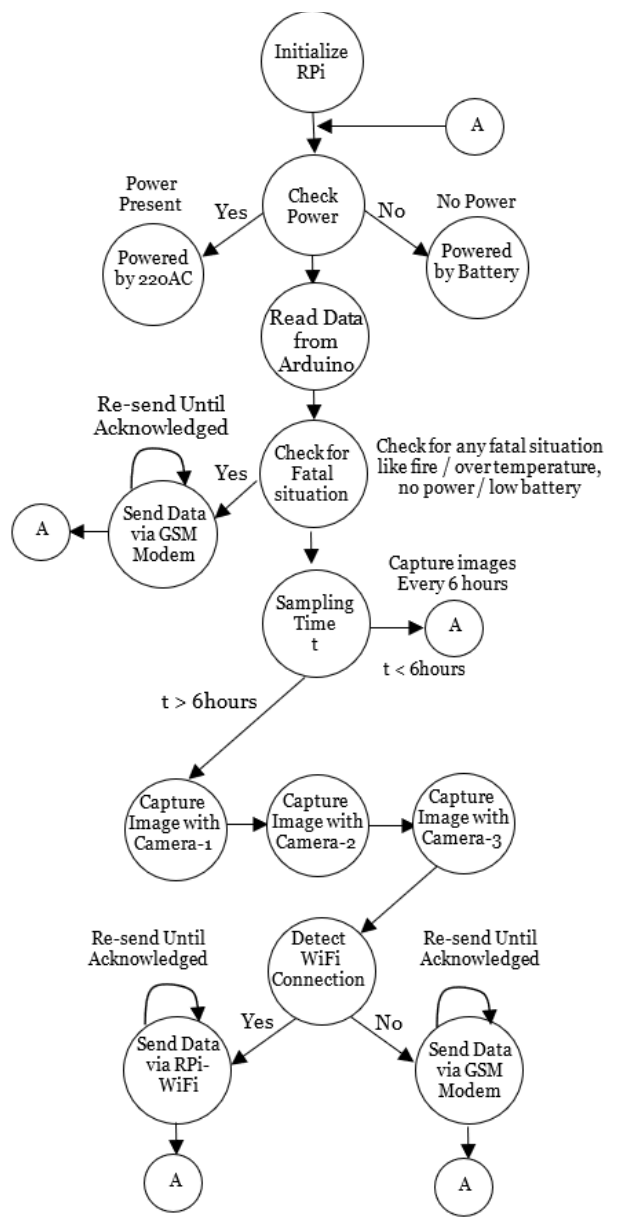

Fig. 8 Overall State Diagram of Software Application for RPi

In the start after initialization of RPi with its OS, it checks status of main supply 220AC and charge-level of battery. If main supply is present RPi switch the whole 
system to take power from it. Similarly, in case of main supply failure, it will switch to the battery $(12 \mathrm{~V}, 12 \mathrm{~A})$ backup, in addition if the battery is not charged enough i.e. below $25 \%$, it will send SOS signal via GSM modem to the concerned user indicating the current situation of power failure with low battery level. If the above stated conditions are satisfactory, the RPi will read data from Arduino-Mega which comprises data from various sensors attached to the system. The data presents overall picture of the laboratory which includes, level of methane gas, fire / smoke detection, temperature, humidity and ambient light intensity. If there is any sign of methane gas or fire and smoke, the RPi will declare fatal situation and send current status of the system to the concerned user or responsible person for initiating remedial action. RPi will keep on checking the fatal situation until it is fixed. In the next stage, the 03nos. of camera images are scanned for capturing images of the explants under observation. The images are captured after every 6 hours or as configured by the user. The sensors-data along with the images-data are transmitted via the RPi$\mathrm{WiFi}$ and insured its proper reception at cloud application, i.e. resend data until acknowledged by cloud application. The overall state diagram is shown in Fig.8

The software application for ArduinoMega monitors the attached sensors via I2C interface. All the sensors incorporated are I2 $\mathrm{C}$ compatible. The sensors are described in detail in the hardware architecture above. The sensors are scanned for every 5 minutes or as configured by the user. The individual sensor's data are aggregated and transformed into a Data-Frame, it is then sent to RPi on its request with insuring proper transfer by receiving acknowledgement from $\mathrm{RPi}$. The Arduino is connected to RPi via USB interface. The state diagram of software application for Arduino is shown in Fig.9.

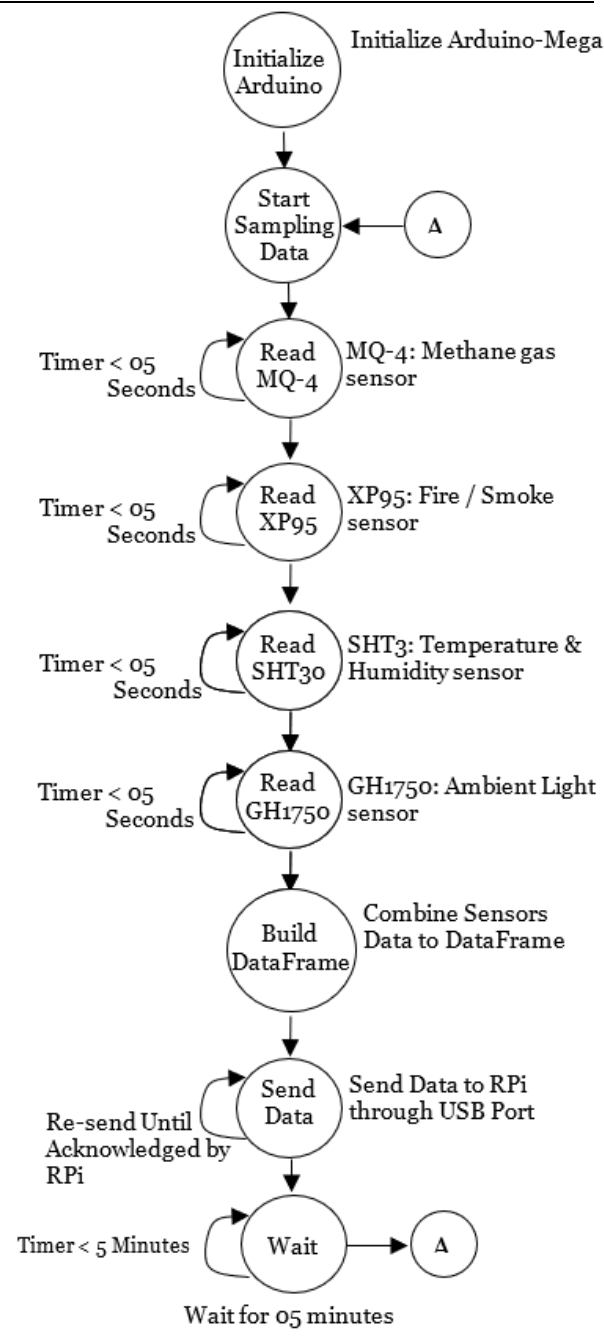

Fig. 9 State Diagram of Software Application for Arduino.

\subsection{Data Formation at Device STCLMS}

The sensors data are transformed to a DataFrame for sending it via GSM-modem or through RPi-WiFi depending availability.

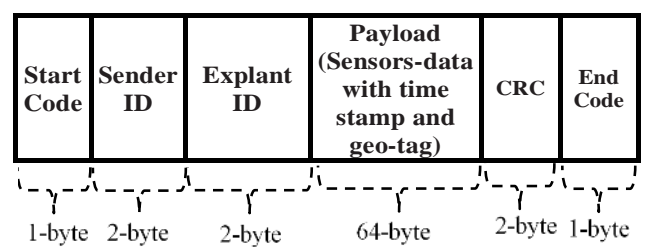

Fig. 10. Data-Frame sends by TCLMS Device to Cloud Application 
Mujahid Hussain Memon et al. Design of a Centralized Intelligent Expert System and Contamination Detection of

The data-frame is comprised of Device-ID Explant-ID, Sensors-Data and CRC for checking frame integrity as shown in Fig. 10.

\subsection{Data-Frame Formation by Cloud Application}

The cloud application performs data analytics, data mining and AI over the logged data. In response it sends alerts and control parameters to a specific TCLMS for smooth running of process in the PTC-growth rooms. The frame format is shown in Fig. 11. It comprises Sender-ID (i.e. Cloud-application), Location-ID (i.e. receiving TCLMS at a particular location) Payload and CRC for checking integrity of frame.

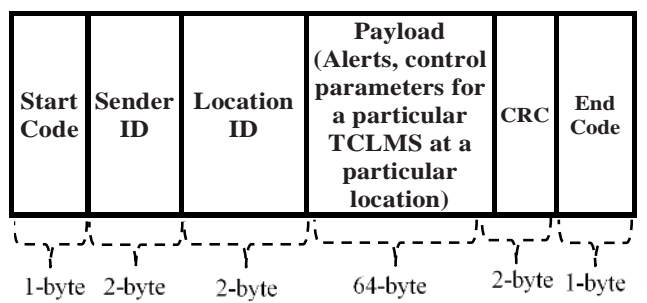

Fig. 11. Data-Frame sends by Cloud Application to TCLMS Device

\subsection{Database Tables for Critical Parameters}

The data collected from different parameters are transmitted to the cloud application and stored in the cloud database. The schema of database comprises three tables, i.e, Expert System Table, Sugarcane Variety Type Tables, and Expert Opinion Table as shown in Table. 1, 2 \& 3 respectively. The Plant Type Table represents the plant names that are under PTC process; it is also labelled, which part of the mother plant is taken for PTC-growth, which may be a piece from root, shoot, leaf or some other part. Similarly, the Expert System Table represents as main table where data of different parameters would be stored. The monitoring, data-analytics and data-mining will help in identifying potential part of plant for PTCgrowth.
TABLE 1: A Sample Database for "Expert System"

\begin{tabular}{|c|c|c|c|c|c|c|}
\hline $\begin{array}{c}\text { Time- } \\
\text { Stamp }\end{array}$ & $\begin{array}{c}\text { Loca- } \\
\text { tion } \\
\text { ID }\end{array}$ & $\begin{array}{c}\text { Plant } \\
\text { ID }\end{array}$ & $\mathbf{T}^{\mathbf{o}} \mathbf{C}$ & $\begin{array}{c}\text { Light } \\
\text { (Hrs) }\end{array}$ & $\begin{array}{c}\text { Light } \\
\text { (Lux) }\end{array}$ & $\begin{array}{c}\text { Humi- } \\
\text { dity } \\
\text { (RH) }\end{array}$ \\
\hline $\begin{array}{c}\text { 01.10.2020: } \\
\text { 11AM }\end{array}$ & 21 & 1 & 25 & 12 & 6500 & 62 \\
\hline $\begin{array}{c}\text { 01. 10.2020: } \\
\text { 01PM }\end{array}$ & 21 & 1 & 24 & 12 & 6450 & 61 \\
\hline $\begin{array}{c}\text { 01. 10.2020: } \\
\text { 08PM }\end{array}$ & 26 & 3 & 27 & 14 & 4500 & 72 \\
\hline I & i & ! & & $!$ \\
\hline
\end{tabular}

TABLE 2: A Sample Database for "Sugarcane Variety Type"

\begin{tabular}{|c|c|c|c|c|c|}
\hline $\begin{array}{c}\text { Plant } \\
\text { ID }\end{array}$ & $\begin{array}{c}\text { Plant } \\
\text { Name }\end{array}$ & $\mathbf{T}^{\mathbf{0}} \mathbf{C}$ & $\begin{array}{c}\text { Light } \\
\text { (Hrs) }\end{array}$ & $\begin{array}{c}\text { Light } \\
\text { (Lux) }\end{array}$ & $\begin{array}{c}\text { Humidity } \\
\text { (RH) }\end{array}$ \\
\hline 1 & $\begin{array}{c}\text { Sugarcane } \\
\text { Variety 1 }\end{array}$ & 24 & 12 & 6000 & 60 \\
\hline 2 & $\begin{array}{c}\text { Sugarcane } \\
\text { Variety 2 }\end{array}$ & 25 & 16 & 7522 & 63 \\
\hline 3 & $\begin{array}{c}\text { Sugarcane } \\
\text { Variety 3 }\end{array}$ & 28 & $12 \sim 16$ & 4500 & 70 \\
\hline i & i & i & i & i & i \\
\hline
\end{tabular}

TABLE 3: A Sample Database for "Expert Opinion"

\begin{tabular}{|c|c|c|c|c|}
\hline Timestamp & ES_ID & $\begin{array}{c}\text { Location } \\
\text { ID }\end{array}$ & $\begin{array}{c}\text { Plant } \\
\text { ID }\end{array}$ & xpert Opinio \\
\hline $\begin{array}{c}01.10 .2020: \\
\text { 11AM }\end{array}$ & 1 & 21 & 1 & Healthy \\
\hline $\begin{array}{c}\text { 01. 10.2020: } \\
01 \mathrm{PM}\end{array}$ & 1 & 21 & 1 & Healthy \\
\hline $\begin{array}{c}\text { 01. 10.2020: } \\
08 \mathrm{PM}\end{array}$ & 2 & 26 & 3 & Healthy \\
\hline i & $\mathrm{i}$ & $\mathrm{i}$ & $\mathrm{i}$ & $\mathrm{i}$ \\
\hline
\end{tabular}

\subsection{Data Formation at Device STCLMS}

There is a huge variety of cloud computing flavors to host the cloud application, but Amazon Web Services (AWS) provided by Amazon is chosen to reduce the cost of software development time for the project. In AWS, the Elastic Compute Cloud (EC2) with Ubunto is customized. It is free 12 Months with hardware configuration of 1-GB RAM and 8GB storage capacity [10]. The collected 
Mujahid Hussain Memon et al. Design of a Centralized Intelligent Expert System and Contamination Detection of

data from edge devices are stored in database (MongoDB) at S3 Bucket.

In order to achieve such goals of real-time AI-Model generation and monitoring its performance, AWS facilitates a comprehensive and fully managed services that covers cloud computing environment including an Instance, Storage, Machine Learning Workflow, Device Integrity, Security and Availability. AWS also provides encryption by default during communication and storage in S3 bucket. The application would be deployed on AWS-EC2 and required micro services including APIs of Application and RPi would be integrated with SageMaker. A microservice will fetche data from and to S3 bucket. To train the model, Amazon provides 'AWS SageMaker' development environment with Notebook IDE and SageMaker Studio; a Program-less environment for training, testing and creating AI Model with readymade algorithms. SageMaker supports deep learning-based AI frameworks which include TensorFlow, PyTorch, Apache MXNet, Chainer, Keras, Gluon, Horovod, Scikit-learn and Deep Graph Library. In addition to this, version control of DNNs Models has been carried out real time so that evaluation of different models be tested and compared.

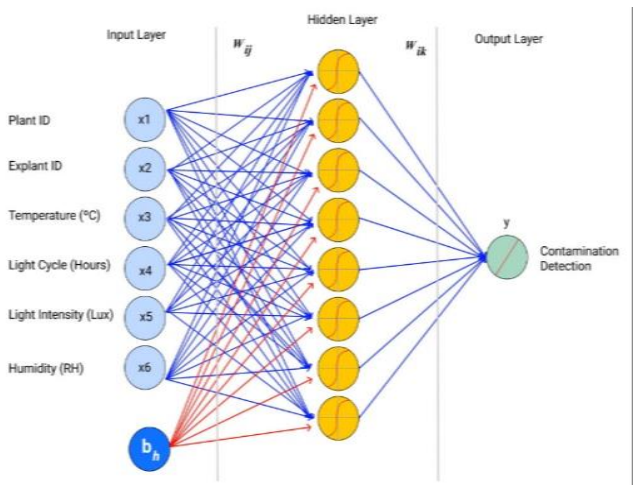

Fig.12. Topology of Developed Neural Network that identifies the detection of contamination i.e. "Explant is in danger"

At initial stage the data was not sufficient for ML/ DL, so a simple rule-based functions was embedded to perform the tasks. After a period of six months when sufficient data was collected on cloud, the AWS Sagemaker was instantiated on the server side as a AI-agent to monitor the critical parameters of explants. For taking timely decisions by the PTC-Experts a Decision Support System (DSS) was incorporated to classify plants as either "Healthy" or "Contaminated" as shown in Fig. 12.

$\mathrm{H}$ To address the contaminated situation when the situation becomes disastrous, AIModel will indicate the alerts i.e. Light Intensity too Low, Light Intensity too high, Temperature too Low, Temperature too High, Light Cycle too Low, Light Cycle too High, so that the corrective procedures can be carried out timely.

In case, when there is a danger situation for the explants, the AI-agent needs to address the issue by identifying potential causes of danger that may have occurred so that the corrective procedures can be carried out. An elaboration of the AI-agent for plant in danger is shown in Fig.13.

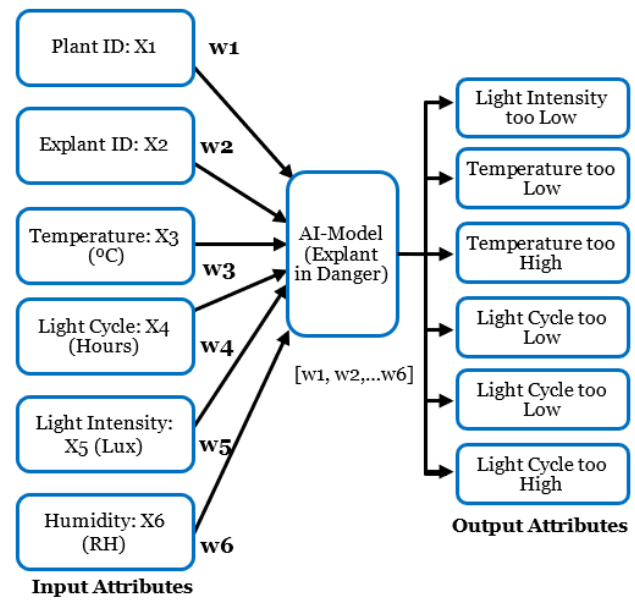

Fig.13. AI-Model identifying potential causes of danger.

"Design of Centralized Intelligent Expert System and Contamination Detection of Tissue Cultured Sugarcane Crop" is based on independent micro-services. To serve Frontend application the visualized and summarized dashboard can be designed in any suitable interface development tool like PHP, ASP.Net or any mobile application environment as well. In this way the remote 
Mujahid Hussain Memon et al. Design of a Centralized Intelligent Expert System and Contamination Detection of Tissue Cultured Sugarcane Crop

clients (devices), PTC-Experts and Developers will work. The overall project structure is elaborated in Fig. 14.

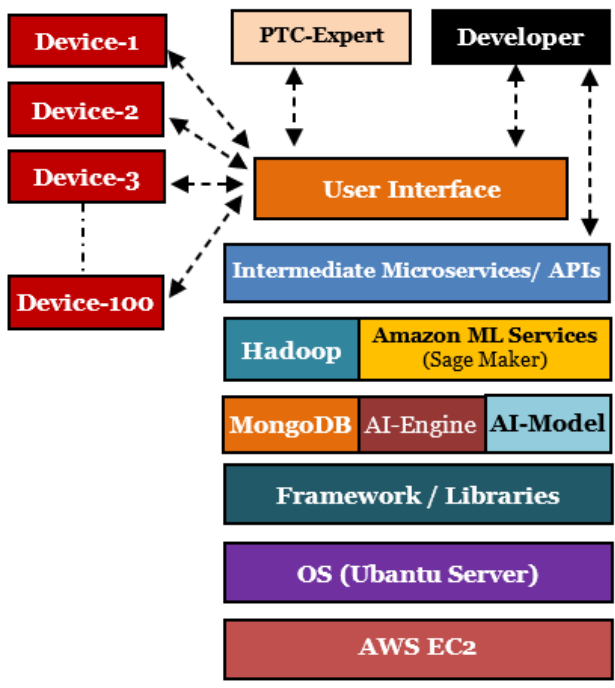

Fig. 14. Cloud Computing architecture based on AWS-EC2

The prediction of explants inside growthroom poses many challenges for big-data analytics and secure IoT infrastructure that needs an appropriate data storage and computational framework with scalable ML environment. Keeping in view data storage, a traditional database (relational database) is inefficient and ineffective to manage big-data. So to achieve the performance and stability with flexible data management system with huge capacity and speed, i.e. NoSQL (MongoDB) was implemented. Additionally, Hadoop data mining was used for decision and proactive analysis which has a significant role to provide services for data-mining.

\subsection{Descriptive to Proactive Approach}

At initial level, after the development of application first the reactive actions are taken; however, as the data gradually increase, the model would be able to perform proactively, for this the steps would be taken Descriptive, Diagnostic, Predictive, Prescriptive and finally Proactive Analysis. In order to train the model with version control on cloud would be required, shown in Fig. 15 Moreover, optionally, a trained AI-Model or a simple Rule-Based algorithm illustrated by PTCexpert can be integrated as well, in order to provide the assistance.

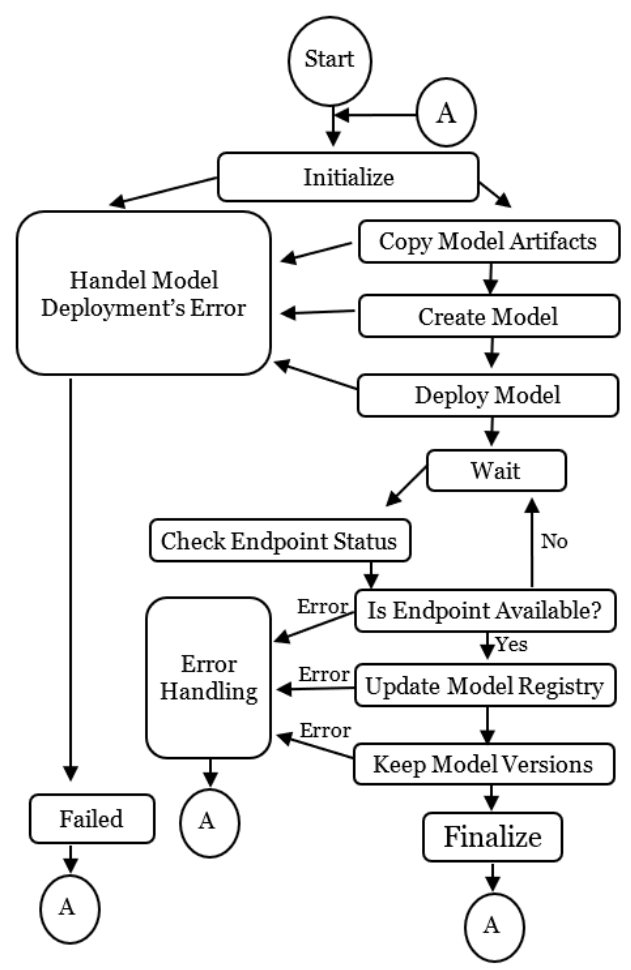

Fig. 15. Steps for AWS SageMaker Training \& Deployment of AI-Model with version control.

he sensors data are transformed to a DataFrame for sending it via GSM-modem or through RPi-WiFi depending availability. The data-frame is comprised of Device-ID Explant-ID, Sensors-Data and CRC for checking frame integrity as shown in Fig. 10.

\section{Experiment and Results}

In our experiment, we used high-resolution images which were captured by our installed webcams inside growth-room where sugarcane crop was cultured. To automate the identification of 'Healthy' and 'Contaminated' tissue cultured plants using deep learning methods on captured pictures of explants, Convolution Neural Networks achieved classification accuracies of $95 \%$ as shown in Fig. 18. The advantage of DL method is also 
Mujahid Hussain Memon et al. Design of a Centralized Intelligent Expert System and Contamination Detection of

being able to automatically learn the features from the input data. The dataset was divided into two sets of $70 \%-30 \%$ size (3500 - 1500) training - testing respectively. Table 4 shows the contaminated (See Fig 16) and healthy (See Fig 17) for training set and testing set.
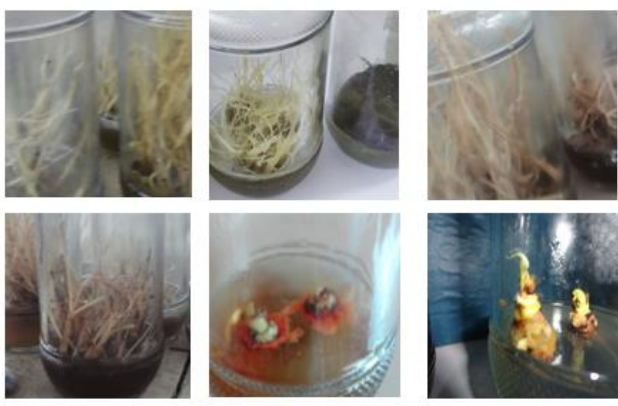

Fig. 16. Images showing the Contaminated Sugarcane Crop
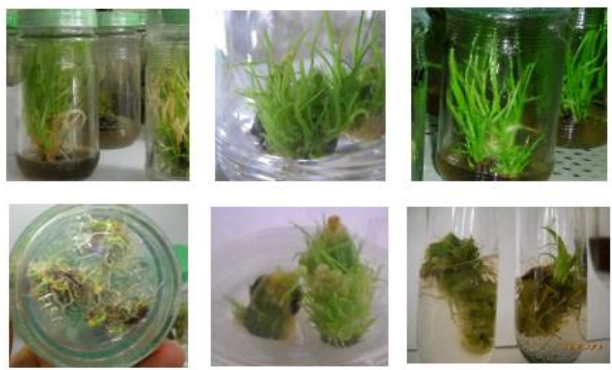

Fig. 17. Images showing the Healthy Sugarcane Crop

TABLE 4: Distribution of two types Healthy and Contaminated explant.

\begin{tabular}{|l|c|c|}
\hline Label & Test Set & Training Set \\
\hline Contaminated & 1750 & 750 \\
\hline Healthy & 1750 & 750 \\
\hline
\end{tabular}

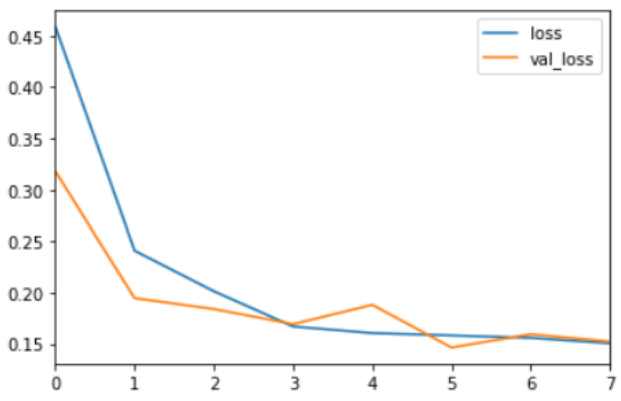

Fig. 18. Graph of training and loss of trained AI Model
TABLE 5: Distribution of two types Healthy and Contaminated explant.

\begin{tabular}{|l|c|c|c|}
\hline Label & Precision & Recall & $\begin{array}{c}\text { F1 } \\
\text { Score }\end{array}$ \\
\hline 0 (Contaminated) & 0.96 & 0.94 & 0.95 \\
\hline 1 (Healthy) & 0.94 & 0.96 & 0.95 \\
\hline Accuracy & & & \\
\hline Macro avg & 0.95 & 0.95 & 0.95 \\
\hline Weighted avg & 0.95 & 0.95 & 0.95 \\
\hline
\end{tabular}

In convolutional neural network Feature Detectors are represented as matrixes which helps to detect the relevant features in a given image. There are many filters for this process but 'sharpen kernel' filter makes the images sharper due to the increase in intensity of pixels as shown in Fig. 19.

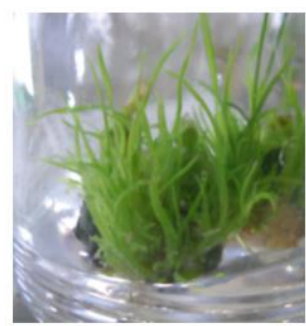

Original Image

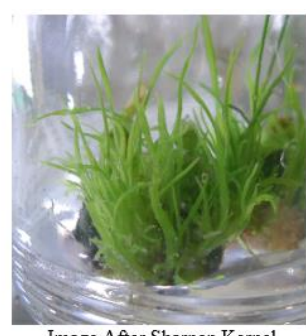

Image After Sharpen Kernel Applied
Fig. 19. Showing before and after results of Feature Detection process step

The modern generation of convolutional neural networks (CNNs) has made excited results in image classification. This approach has been used to develop the detection of tissue cultured plant for recognition of contaminated and healthy growth by the use of Deep Convolutional Neural Network. The ADAM optimizer is used which is well in practice. We preprocessed the data by subtracting the average RGB value from our real 5000 captured images of sugarcane cultured plant is $1000 \mathrm{px}$ by $1000 \mathrm{px}$ resolution. In order to gain better comparison with advanced methods, we used the parameters of "Healthy" and "Contaminated" for the training-set and testing-set, which are commonly adopted. However the image size were reduced to $500 \mathrm{px}$ by 500px to decrease the training time. The proposed model was trained on HP Z620 Dual Processor Intel Xeon E5-2640 Dual HexaCore Processor via NVIDIA GTX 1660ti 
Mujahid Hussain Memon et al. Design of a Centralized Intelligent Expert System and Contamination Detection of

4GB DDR5 GPU, which took nearly 2 hours in 250 epochs. It is implemented in KERAS python 3.5.6. The output of neuron is 0 or 1 , the captured pictures were also cropped manually so the unnecessary objects may not come under the classification and better result be achieved. The classification after training was made and the results were achieved as shown in Table 5.

In order to improve the resolution of pictures, so the detection of contaminated and healthy plants could be identified truly, the Generative Adversarial Networks (GANs) was applied. The impressive performance was reported with the existing deep learning-based super resolution methods; however, the limitations were also notified to overcome this challenge. The architectural design of Deep Neural Network models are susceptible to make small changes, where it may generate a contaminated high-resolution (HR) picture of an healthy plant or its inversely, it may generate a picture with more contamination of any plant that may have a partial contamination and could survive inside the media. It is the Residential Block that may weakens the network as well. In order too overcome this critical situation, we have carefully studied the core problem of Super Resolution Models where residential we have applied wide residual network [25]. To address these issues, we have worked on SuperResolution GANs (SRGAN), The residual block is the building block of the Residual Network (ResNet), which is one of the top architectures used for Image Recognition. In this technique we increase the width and reduce the depth of the residual network, due to decreasing the depth of the network our model reduced memory costs. However, bicubic interpolation method is also widely used as the input image, which is expensive in terms of memory and needs additional computation.

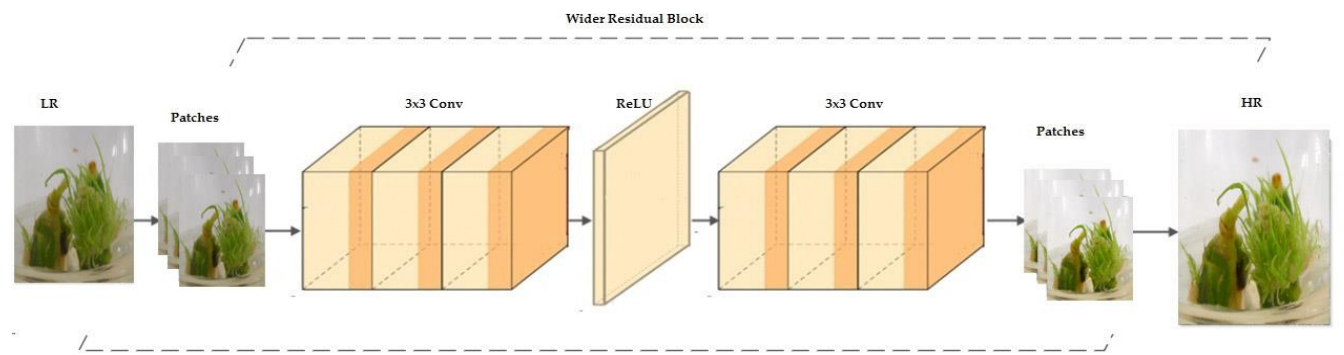

Fig. 20. Figure showing proposed Wider Residual block which has $(2 \times$ to $4 \times)$ slim identity pathway channels before activation in each residual block

As we have applied wide residual network architecture to improve the performance of residual network significantly. To resolve the problem of over-fitting of model in the residual system, we have used extended weigh normalization.

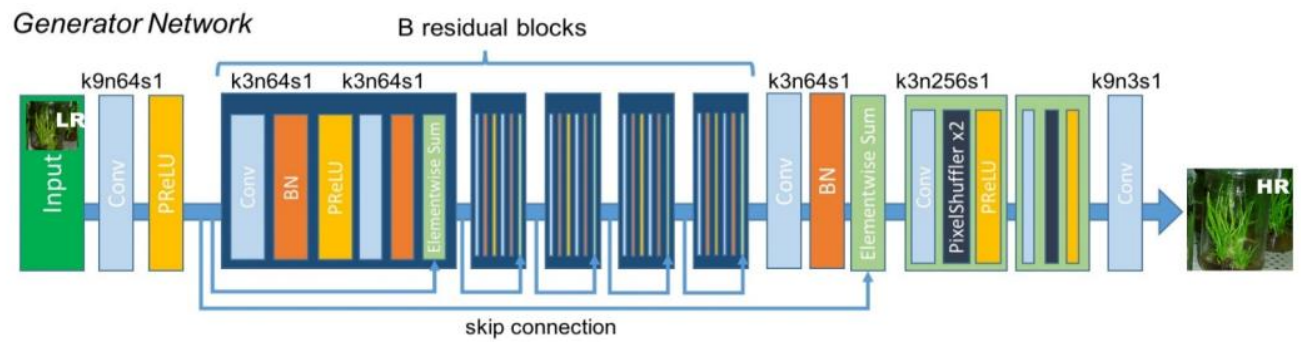

Fig. 21. Architecture shows Generator Network consisting kernel size (k), number of feature maps (n) and stride (s) designated for each Conv layer 


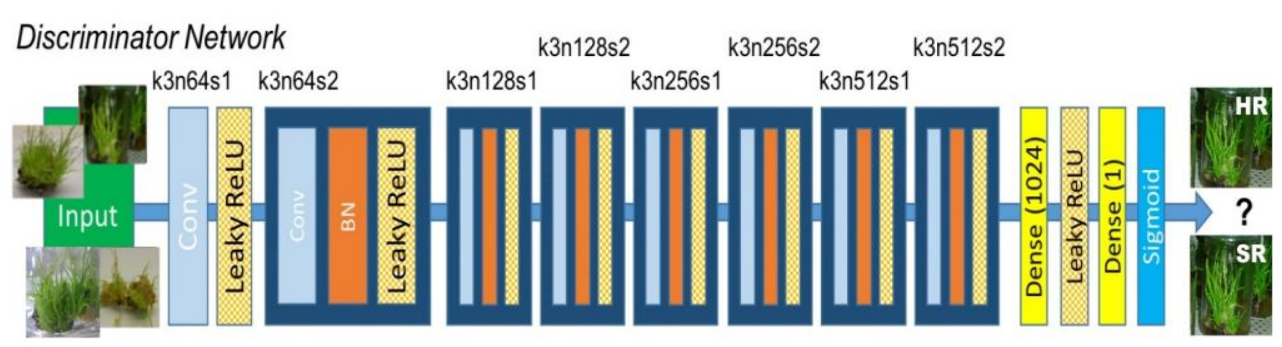

Fig. 22. Architecture shows Discriminator Network consisting kernel size (k), number of feature maps (n) and stride (s) designated for each Conv layer

In our experiment we have used multiple images of same direction from a dataset. To measure the Peak Signal to Noise Ratio (PSNR) in RGB space, the ADAM optimizer was also used. The experiential results demonstrate that Adam Optimizer worked well; moreover, it has a great advantage over other methods like stochastic optimization. We also used a random horizontal flip and a 90-degree rotation to crop 56x56 RGB LR input images from HR images.

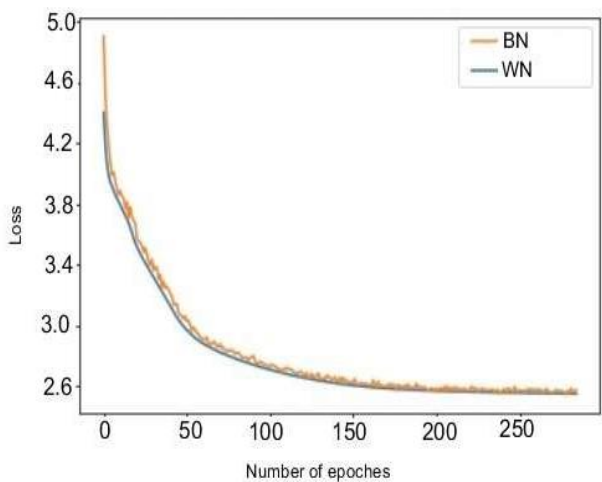

Fig.23. Training L1 loss of our model trained with batch and weight normalization

The proposed SRGAN model was trained on a $3.20 \mathrm{GHz}$ CPU via the NVIDIA GTX1650 Super GPU, which took nearly 24 hours in 250 epochs. It is implemented in KERAS python 3.5.6. It has been compared with the existing bicubic interpolation method.
TABLE 5: Shows the results of applied SRGAN for image enhancement.

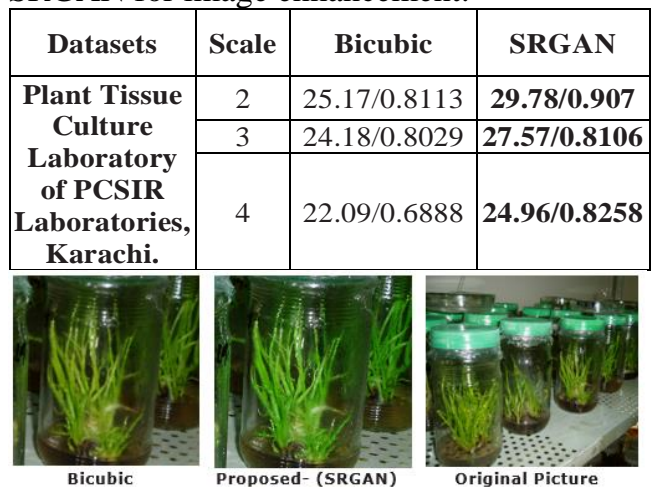

The goal of generator is to create images for discriminator. It just simple generate the noise from also grab pictures from real dataset. At the stage of training of discriminator, we label the fake generated images as zero and real as one. So, as time goes, the second stage of training is going to keep improving and generates the images trying to improve the quality of picture, unless the picture becomes able to be recognized in better quality than previous one. Meanwhile, the picture is also compared with the real dataset, so there be no difference between false and real images.

It is the beauty of GANs, the generator never gets to see the actual real images, it generates convincing images only based off gradients flowing back through the discriminator. In addition to this, the discriminator improves as training phases continuing, means the generated images will also need to get better and better. 


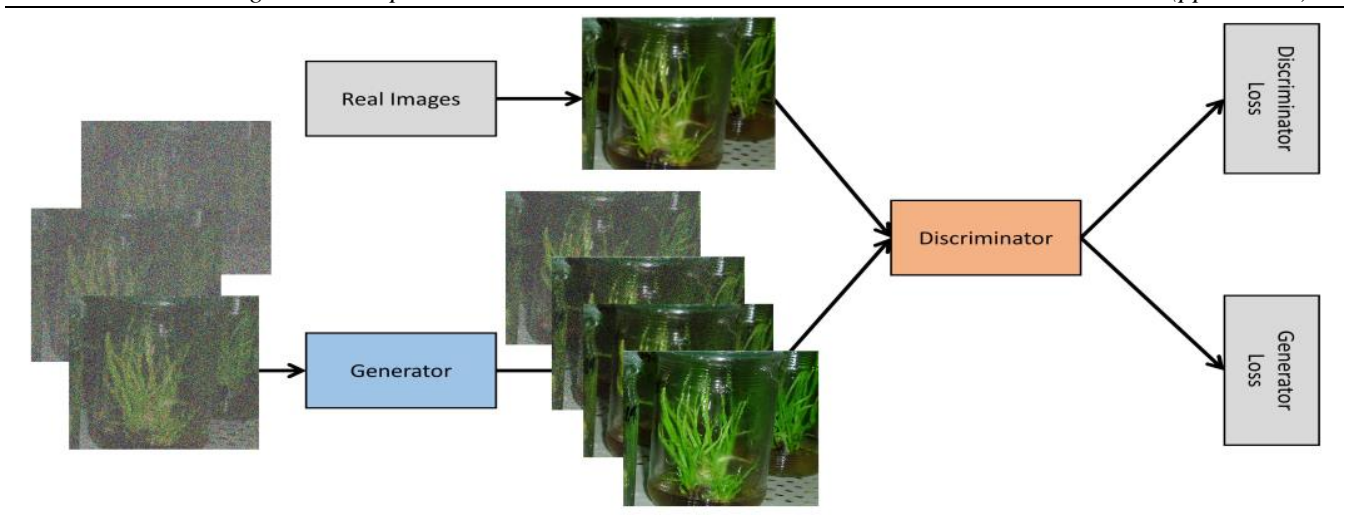

Fig. 24. Model Shows Architecture of GANs applied on Sugarcane Crop

\section{Conclusion}

The Design of Centralized Intelligent Expert System and Contamination Detection of Tissue Cultured Sugarcane Crop; for monitoring the production inside laboratory / growth-rooms has been presented. It monitors essential parameters such as temperature, humidity, light intensity, flammable gases and fire / smoke. In addition, 3nos. of webcams are attached for visual monitoring of explants and growth rooms. The images along with data are transmitted to the cloud services of AWS, where the analytics, data mining and AI decisions are taken. This designed is intended to be installed at around 100 growth rooms in several areas of country, so that PTC-experts can monitor different growth-room across the country which would otherwise be impossible to travel and visit timely. Moreover, the proposed model incorporates Convolutional Neural Network (CNN) and Generative Adversarial Networks (GAN)" for detection of contamination and enhancement of image quality at edge device respectively, in this way GAN will provide High Resolution (HR) images, i.e. Single Image Super Resolution (SISR) [26] [27]. The edge device is based on RaspberryPi-4 and Arduino Mega, making the connectivity via RPi-WiFi or GSM-modem (Sim900). The core purpose of system is to identify any type of contamination inside the growth-room and health of explants with detecting any disastrous situation like firesmoke or flammable gas for fire protection.
The data collected at the cloud application will enable data mining, data analytics and AI which will help PTC-experts for proactive measures and future decision making to increase crop yield and quality, thus leading to coping the challenge of food scarcity and insecurity of next era.

\section{REFERENCES}

[1] K. K. Behera and S. Sahoo, "Rapid In vitro Micro propagation of Sugarcane (Saccharum officinarum L. cvNayana) Through Callus Culture," Nature and Science, vol. 7 , no. 4, pp. 1545-0740, 2009.

[2] R. H. Smith, Plant Tissue Culture: Techniques and Experiments, Academia Press of Elsevier , 2013.

[3] B. Getnet, "Review on In Vitro Propagation of Sugarcane to," Agricultural Research \& Technology Open Access Journal, vol. 5, no. 4, 2017.

[4] C. Mohan, Sugarcane Biotechnology: Challenges and Prospects, Cham: Springer, 2017.

[5] B. D and T. T, "Organization of a plant tissue culture laboratory. In: (ed.) Vasil I. Cell culture and somatic cell genetics of plants" in Laboratory procedures and their applications, New York, Acadademy Press, pp. 1-12, 1984.

[6] G. O. L, M. T, T. T. A and V. I.K, "Plant tissue culture. In vitro cellular and 
developmental biology," Physiological Plant, vol. 12, pp. 473- 478, 2009.

[7] F. Jeff, P. Gerald and P. Raul , "Comparison of sugarcane disease incidence and yield of field-run, heattreated, and tissue-culture based seedcane," Journal of American Society of Sugar Cane Technologists, vol. 25, pp. 88-100, 2005.

[8] S. Nandita , K. Anil and K. G. Girish , "Genotype dependent influence of phytohormones combination and sub culturing on micro propagation of sugarcane varieties.," Indian Journal of Biotechnology , vol. 5, no. 1, pp. 99-106, 2006.

[9] J. N, N. D and S. T, "Micropropagation for Quality Seed Production in Sugarcane in Asia and the Pacific," USA, Food and Agriculture Organization of the United Nation, pp. 13-60, 2008.

[10] S. K, R. Sengar and S. Garg, "The effect of in vitro environmental conditions on some sugarcane varieties for micro propagation," African Journal of Biotechnology, vol. 10, no. 75, pp. 1712217126, 2011.

[11] S. Snyman, G. Meyer, J. Richards, S. Ramgareeb and . B. Huckett, "Refining the application of direct embryogenesis in sugarcane: effect of the developmental phase of leaf disc explants and the timing of DNA transfer on transformation efficiency," Plant cell reports, vol. 25, no. 10, p. 1016-1023, 2006.

[12] S. Sandhu, S. Gosal, K. Singh, G. Cheema and M. Meeta , "Field performance of micro propagated plants and potential of seed cane for stalk yield and quality in sugarcane.," Journal of Sugar Technology, vol. 11, no. 1, pp. 34-38, 2009.

[13] A. Kuar and . S. Sandhu, "High throughput in vitro micro propagation of sugarcane (Saccharum officinarum L.) from spindle leaf roll segments: Cost analysis for agri-business industry.," Plant Cell Tissue Organ Culture, vol. 120, no. 1, pp. 339-350, 2014.

[14] C. R, Plant microbiology, London: Edward Arnold Ltd, 1985 .
Microbial Contamination Management in Micropropagation, Netherlands: Springer , 1997

[16] P. G. Taherei , H. D. Hossein and A. Mosavi, "Sugarcane growth prediction based on meteorological parameters using extreme learning machine and artificial neural network," Engineering Applications of Computational Fluid Mechanics, vol. 12, no. 1, p. 738-749, 2018.

[17] A. K. Aman Jain, "Smart Agriculture Monitoring System using IoT," International Journal for Research in Applied Science \& Engineering Technology, 2020.

[18] D. U. D. a. N. P. Sobejana, "Automated relative humidity and temperature control system for banana tissue culture laboratory with monitoring system and SMS notification," International Journal of Engineering in Computer Science, 2019.

[19] A. A. Elsh `arif and S. S. Abu-Naser, "An Expert System for Diagnosing Sugarcane Diseases," International Journal of Academic Engineering Research, vol. 3, no. 3, pp. 19-27, 2019.

[20] N. H. A. A. a. e. all., "Real Time Monitoring Critical Parameters in Tissue Culture Growth Room with SMS Alert System," IEEE International Conference on Intelligent Systems, Modeling and Simulation, 2010.

[21] B. A. a. e. all, "Using Deep Learning to Predict Plant Growth and Yield in Greenhouse Environments," arXiv preprint arXiv:1907.00624, 2019.

[22] R. N. M. T. \&. M. Y.-N. Mohsen Hesami, "Development of support vector machine-based model and comparative analysis with artificial neural network for modeling the plant tissue culture procedures: effect of plant growth regulators on somatic embryogenesis of chrysanthemum, as a case study," Plant Methods, 2020.

[23] S. P. a. e. al., "Prediction of chemical composition for callus production in Gyrinops walla Gaetner through machine learning," Information Processing in Agriculture, 2019.

[15] Cassells and A. C, Pathogen and 
Mujahid Hussain Memon et al. Design of a Centralized Intelligent Expert System and Contamination Detection of Tissue Cultured Sugarcane Crop

[24] A. Chlingaryan, S. Sukkarieh and B. Whelan, "Machine learning approaches for crop yield prediction and nitrogen status estimation in precision agriculture: A review," Computers and Electronics in Agriculture, vol. 151, pp. 61-69, 2018.

[25] Z. Sergey and K. Nikos, "Wide residual networks," 23 May 2016. [Online].

[26] S. Zhang, G. Liang and S. Pan, "A Fast Medical Image Super Resolution Method Based on Deep Learning Network," IEEE Access, pp. 12319 - 12327, 2018.

[27] Y. "Image super-resolution: The techniques, applications, and future," Signal Processing, vol. 128, p. 389-408, 2016. 